\title{
The Chemistry of Solid-State Electronics
}

\author{
E. YABLONOVITCH
}

Since the original theoretical insights of Bardeen and Shockley about 40 years ago, the progress of solid-state electronics has been paced by the ability to control chemical bonding structures, particularly at surfaces and interfaces. The functioning of solid-state devices depends on being able to produce interfacial structures with a minimum number of defective chemical bonds. A series of chemical discoveries and insights, on germanium (Ge) and silicon ( $\mathrm{Si}$ ) surfaces and gallium arsenide-aluminum arsenide (GaAs-AlAs) interfaces, has brought the electronics revolution to its present state of development. In most cases, the technological consequences of these accidental discoveries could not be accurately foreseen. With that caution, the technological prognosis for some current research is also reviewed.

S EMICONDUCTOR MATERIALS HAVE BECOME SO COMMONplace that we now take them for granted, but they are actually rather exceptional substances. If they were to be discovered all over again in a single year, the public interest and the attendant publicity would be enormous. The advancement of semiconductor electronics had to wait for the discovery of a few lucky chemical coincidences that nature was kind enough to place in our path. Very specific inorganic chemical-bonding structures are required for solid-state electronic devices to function.

\section{Semiconductors as Very Large Molecules}

In chemical terms, what makes a semiconductor crystal so special is that the entire semiconductor crystal is a giant covalent molecule. In benzene, the electron wave functions are delocalized over the six carbon atoms of the ring. In a large dye molecule, these wave functions might be delocalized over a series of rings. But in semiconductors, the electron wave functions are delocalized, in principle, over an entire macroscopic crystal. Because of the large spatial extent of these wave functions, no single atom can have much effect on the electron energies. We say that electronic excitations in semiconductors are delocalized.

Furthermore, good semiconductors are drawn from the central columns (III, IV, and V) of the periodic table, and they tend to be nonpolar. Consequently, the electron-lattice interaction is very weak. In molecular terms, the electronically excited potential energy surface for atomic motion is almost identical to the ground potential energy surface. Such electrons rarely excite vibrational motion in the

The author is a member of the technical staff of the Solid-State Science and Technology Research Laboratory of Bell Communications Research, Navesink Research \& Engineering Center, Red Bank, NJ 07701-7040. solid. As a result, carriers move as if in free space, colliding with the atomic lattice rather infrequently.

Not only do the electrons in the valence and conduction bands (bonding and antibonding orbitals) tend to ignore the crystallographic lattice of atoms, they tend to ignore one another. Electrons excited into the conduction band tend to stay in the conduction band, returning only slowly to the valence band. The corresponding missing electrons in the valence band, called holes, tend to remain in the valence band. The conduction band electrons can establish equilibrium on their own, at their own chemical potential, and electrons in the valence band can have their own equilibrium at a second, different, chemical potential. Instead of having one single chemical potential (or Fermi level) for all the electrons in the material, the possibility exists for two separate quasi-Fermi levels in the same crystal.

The idea of having two distinct quasi-Fermi levels or chemical potentials within the same volume of material, first emphasized by Shockley (1), is a rather striking one. It has deeper implications than the somewhat similar concept of two distinct effective temperatures, which can occur, for example, in nuclear spin systems that have weak spin-lattice coupling. The semiconductor energy level structure is illustrated in Fig. 1, where $E_{\mathrm{Fc}}$, is the quasi-Fermi level or chemical potential of electrons in the conduction band and $E_{\mathrm{Fv}}$ is the quasiFermi level for electrons in the valence band. We can immediately understand two critical opto-electronic devices from Fig. 1:

1) Solar cells: The difference of chemical potentials $E_{\mathrm{Fc}}-E_{\mathrm{Fv}} \equiv \mathrm{eV}$ is the available output voltage $\mathrm{V}$ of a solar cell. Photons create the chemical potential difference simply by boosting a population of electrons from the valence band into the conduction band.

Fig. 1. The energy-level structure of a semiconductor crystal plotted versus electron wave vector $\mathbf{k}$. The upper levels are the conduction band and the lower levels are the valence band. The electrons in each band are somewhat independent, and each band can establish quasi-equilibrium at its own chemical potential (or quasi-Fermi level). Shown is a population inversion, which can lead to optical gain and possible lasing. Conversely, the chemical potential difference $E_{\mathrm{Fc}}$ $-E_{\mathrm{Fv}}$ can be the output voltage of a solar cell. Equilibrium is reestablished by stepwise recombination at defect levels $\mathrm{D}$ within the forbidden gap.

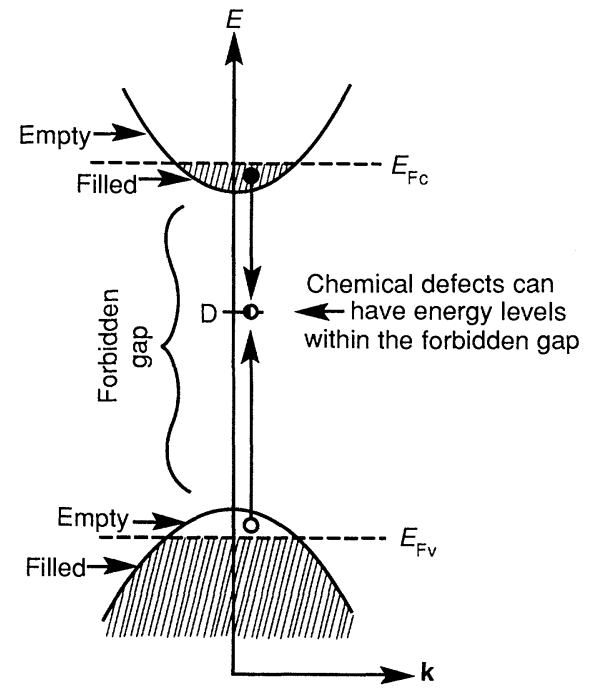

ARTICLES 347 
2) Lasers: The valence band states above $E_{\mathrm{Fv}}$ are essentially (2) empty, and the states below $E_{\mathrm{Fc}}$ are essentially filled. Directly and naturally this permits a population inversion, which is the principle by which lasers operate.

These two functions are the inverse of one another: the semiconductor laser converts electricity into light, and the solar cell converts light into electricity.

The possibility of two separate electronic equilibria (quasi-Fermi levels) requires a very slow decay of electrons from the conduction band back into the valence band (electron-hole recombination). Again, the very weak electron-lattice coupling resulting from the large delocalized wave functions in nonpolar materials is important here. Recombination requires getting rid of the band-gap energy of $\geqslant \mathrm{l} \mathrm{eV}$. If this were to occur as nonradiative processes involving lattice motion, 20 or 30 phonons would have to be emitted. The weak electron-lattice coupling makes this an extremely unlikely (actually vanishingly improbable) event. In contrast, in organic molecules decay by nonradiative recombination is sufficiently likely to occur that it is given the name "internal conversion."

In fact, nonradiative recombination does occur in semiconductors, but primarily as a result of chemical defects that introduce new energy levels into the forbidden gap. These defect levels act as stepping stones, permitting conduction electrons to cascade down to the valence band in two steps, emitting smaller numbers of phonons in each step rather than many more in one improbable giant leap. This model of stepwise nonradiative recombination through impurities or other defects is associated with the names of Hall (3), and Shockley and Read (4). The defects act as electron-hole recombination catalysts, promoting recombination without being consumed themselves.

Thus a major goal of semiconductor materials science has been to create chemically perfect semiconductor structures. Any defects that could disturb the perfect valence bonding structure and allow energy levels to appear in the forbidden gap must be eliminated as far as possible. Although this explains the need for the utmost extrinsic chemical purity, even chemical purity is insufficient. Any intrinsic defects in the most general sense, including broken bonds, selfinterstitials, and vacancies, are also proscribed. In particular, unsaturated chemical bonds on the surface or in the bulk will contribute nonbonding orbitals with unwanted energy levels in the forbidden gap. Fortunately, the rigid, tetrahedrally coordinated semiconductor crystal structures have a tendency to reject both extrinsic and intrinsic defects, which is yet another reason for their technological success.

\section{Chemical Bonding at Semiconductor Surfaces}

Semiconductor surfaces are the most likely location for nonbonding or weakly bonding orbitals to occur. From the very beginning of the semiconductor age, the ability to control chemical bonding structures, particularly at surfaces and interfaces, has paced the progress of solid-state electronics.

The need for surfaces of extremely high chemical quality can be appreciated from Fig. 2A, an original drawing (1) of the first transistor, the point-contact transistor. The emitter point contact emits a cloud of minority carriers (by definition the least dense of the two types, either electrons or holes). These minority carriers undergo a random walk as they try to find the small-area collector point contact (Fig. 2B). If the transistor is to have any significant gain, almost all the minority carriers must be collected. During their random walk, these carriers must pass a gauntlet of defects (labeled $\mathrm{D}$ in Fig. 2B) that could catalyze their recombination.

Specifically, an average carrier scatters off the surface thousands of times before it finally stumbles its way into the collector. If even a tiny fraction of the surface were covered with defects, the carriers would never make it to the collector. It is entirely fortuitous that a Ge surface covered with its natural air-grown oxide has less than 1 part per 10,000 of defective chemical bonds on the surface. The unexpected discovery of the Ge point-contact transistor was therefore critically dependent on this accidental chemical property. The experiment would never have succeeded with Si or with any of the semiconductor compounds used today, as the density of chemical defects at interfaces with their air-grown oxides is just too high.

The bulk chemical defect densities that can be tolerated in solidstate electronics range from 1 in $10^{6}$ to 1 in $10^{11}$, depending on the specific application. Corresponding surface defect densities that can be tolerated range from 1 in $10^{4}$ to 1 in $10^{7}$. All of the semiconductor surface atoms must be cleanly saturated with strong covalent bonds, since weak or strained bonds or nonbonding orbitals could introduce energy levels into the forbidden gap. These requirements on semiconductor surfaces and interfaces give rise to a chemical figure-of-merit: We require a surface chemical reaction with $99.99 \%$ to $99.99999 \%$ yield, which is difficult to achieve. The surface of Ge is unique in that it minimally satisfies this requirement automatically in air. This unanticipated and atypical property permitted the discovery of the first transistor, but the Ge surface could never be further improved.

\section{The $\mathrm{Si}-\mathrm{SiO}_{2}$ Interface}

Beginning in the mid-1950s, Atalla et al. (5) began work on the thermal oxidation of $\mathrm{Si}$. The oxidation recipe was gradually perfected by Deal, Grove, and many others (6). Through trial and error, the figure-of-merit for the $\mathrm{Si}-\mathrm{SiO}_{2}$ interface had been improved to one defective chemical bond in $10^{6}$ by the late 1960s. [The implications were not lost on Grove, who immediately wrote a book (7) and
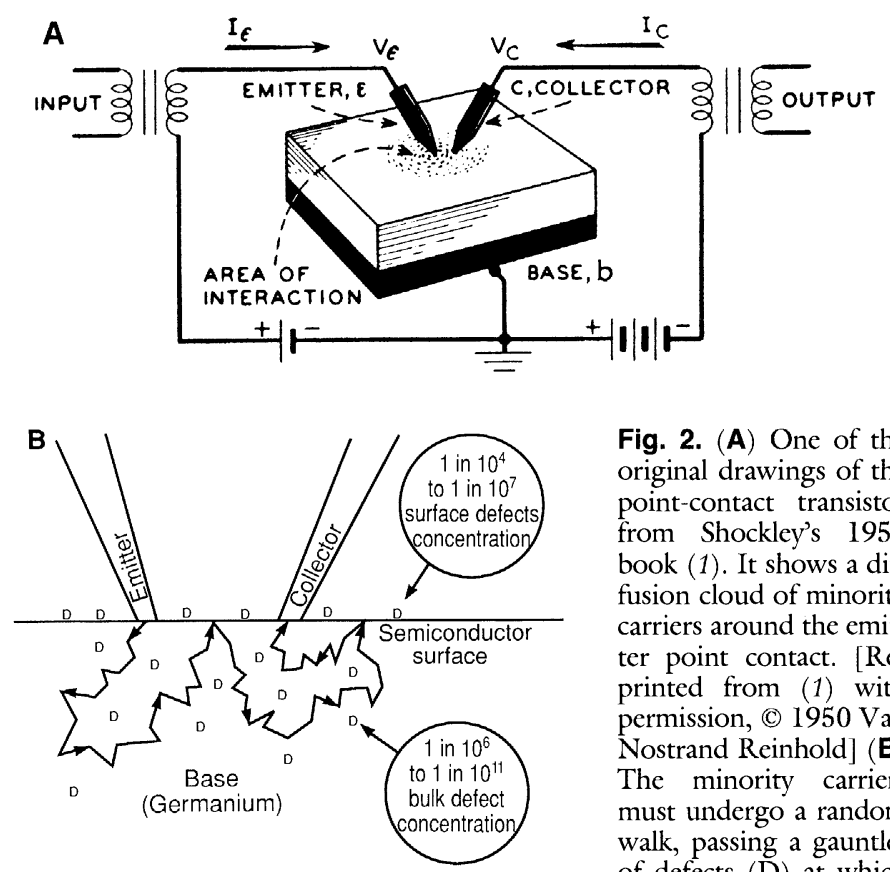

Fig. 2. (A) One of the original drawings of the point-contact transistor from Shockley's 1950 book (1). It shows a diffusion cloud of minority carriers around the emitter point contact. [ $\mathrm{Re}-$ printed from (1) with permission, (C) 1950 Van Nostrand Reinhold] (B) The minority carriers must undergo a random walk, passing a gauntlet of defects (D) at which they could recombine, before perhaps finding

their way to the collector point contact. In practice, the surface concentration of defective chemical bonds must be less than 1 in 10,000 for the carriers to have significant probability of finding the collector contact before recombining. 
Fig. 3. The lattice-matched double heterostructure. The chemical bonds, shown as short horizontal stripes at the interface, match up almost perfectly $(99.999 \%$ saturated). The wave function $\psi(x)$ is sandwiched in at the potential barrier formed by the AlAs and is never exposed to the defective bonds of an external surface. When the GaAs layer is made very narrow, the potential well becomes a quantum well.

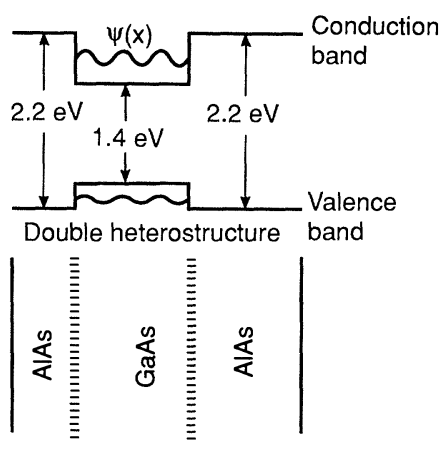

became a founding partner in INTEL.] Although 20 years had already passed since the discovery of the original transistor, in short order there arrived the microprocessor (1969), the memory chip (1971), and the pocket calculator (1972). At present, microchip production is a $\$ 40$-billion industry that supports a $\$ 500$-billion systems, software, and communications industry. All of this has been made possible by the favorable chemical bonding structure of a specially prepared, thermally oxidized $\mathrm{Si}-\mathrm{SiO}_{2}$ interface.

This amorphous-crystalline heterojunction is the most important material system of our era. The interfacial bonds can be $99.9999 \%$ saturated. After 20 years of investigation there is still no widely accepted explanation for this remarkable property.

Chemistry is as important in the bulk as on the surface. As before, the goal is structured perfection and chemical purity. Semiconductor $\mathrm{Si}$ is purified by the distillation of $\mathrm{SiHCl}_{3}$ followed by chemical vapor deposition (CVD) of bulk polycrystalline $\mathrm{Si}$ :

$$
\mathrm{SiHCl}_{3}+\mathrm{H}_{2} \stackrel{1100^{\circ} \mathrm{C}}{\longrightarrow} \mathrm{Si} \downarrow+3 \mathrm{HCl} \uparrow
$$

Purified polycrystalline CVD Si from this reaction is then melted, and a single crystal boule weighing as much as $50 \mathrm{~kg}$ is pulled from the melt by Czochralski growth. Of the two processing steps, CVD and crystal growth, CVD is the more expensive. It is slow, taking as long as 2 weeks, and is therefore very capital intensive. During that long period, heat is continually radiated away at $1100^{\circ} \mathrm{C}$, making it very energy intensive as well. The single crystal boule produced by these processes can have a bulk purity of one part in $10^{11}$, not counting $\mathrm{O}$ and $\mathrm{C}$ impurities, which are relatively benign. Normally the entire boule is structurally perfect, that is, it has zero dislocations. This means that the atomic positions are in registry from one end of the sausage-shaped boule to the other; virtually every atom is in its place. Worldwide, $\geq 30,000$ metric tons of this substance are produced per year. The top grade of semiconductor silicon may be the most perfect material in the universe, with the possible exception of neutron stars; nevertheless the cost is $<\$ 5$ per wafer $(10-\mathrm{cm}$ diameter).

\section{III-V Interfaces}

For opto-electronics, the binary compound semiconductors taken from columns III and V of the periodic table are essential. They often have direct rather than indirect band gaps, which means that, unlike $\mathrm{Si}$ and Ge, their lowest-lying absorption levels interact strongly with light. The basic devices of optical communications, light-emitting diodes (LEDs) and semiconductor lasers, are made of III-V semiconductors. GaAs, AlAs, and their alloys have historically been the most important III-V material system. The reason once again derives from the need to control interfacial chemical bonding structures. The double heterostructure (Fig. 3), invented (8) in the early 1960s, is a crystalline "sandwich" with the "bread" made of
AlAs and the "filling" made of GaAs. Since the band gaps of AlAs and $\mathrm{GaAs}$ are $2.2 \mathrm{eV}$ and $1.4 \mathrm{eV}$, respectively, the $\mathrm{GaAs}$ wave functions $\psi(x)$ are "sandwiched in" by potential barriers (Fig. 3). Although they are prevented from seeing any external surface, they do see the AlAs-GaAs interface, and it is here that nature provides another remarkable accident. The cubic unit cell dimensions of GaAs and AlAs are $5.6533 \AA$ and $5.6605 \AA$, respectively. The mismatch is less than $0.1 \%$, which means that the crystal structures can match up nearly perfectly, leaving only a few unsaturated chemical bonds at the interface. Generally, the interfacial bonds in the $\mathrm{Ga}_{1-x} \mathrm{Al}_{x} \mathrm{As}$ system are $99.999 \%$ saturated, which is not as good as the best Si$\mathrm{SiO}_{2}$ interfaces, but excellent nonetheless.

When the sandwich in Fig. 3 is made very narrow, quantum confinement changes the wave functions $\psi(x)$ in the GaAs. Excellent control is possible because the layers can be grown one monolayer at a time (9). The wave functions can also be modified by alloying Ga or In (or both) into the AlAs. Moreover, As can be substituted by $\mathrm{P}$ or $\mathrm{Sb}$. Furthermore, we have learned how to impose lattice matching by intentionally straining mismatched systems (10). Today, many researchers are working toward extending these artificial structures into the third dimension (11). As a result, we now do "band-structure engineering" and make "designer wave functions."

It used to be a truism that organic chemistry was richer than inorganic chemistry because it offered the possibility of so many more different molecular structures. In fact, the single giant molecule that is the inorganic semiconductor crystal has innumerable possible arrangements, far greater than those which occur in typical organic molecules. With respect to the call for a "molecular electronics" based on organic (12) molecular structures, we are already making quantum structures from semiconductors with features comparable in size to organic molecules. Hydrocarbon-based molecules do not satisfy the requirements of wave function delocalization, weak vibronic coupling, and structural perfection to the same degree that the successful inorganic semiconductors do.

One can contrast the physics and chemistry approaches to III-V synthesis and epitaxial growth (growth in perfect registry with the atoms of an underlying crystal). The physics approach is molecular beam epitaxy (MBE), which is essentially the evaporation of the elements. The chemistry approach is organometallic chemical vapor deposition (OMCVD), which is exemplified by the following typical chemical reaction:

$$
\mathrm{Ga}\left(\mathrm{CH}_{3}\right)+\mathrm{AsH}_{3} \stackrel{580^{\circ} \mathrm{C}}{\longrightarrow} \mathrm{GaAs} \downarrow+4 \mathrm{CH}_{4} \uparrow
$$

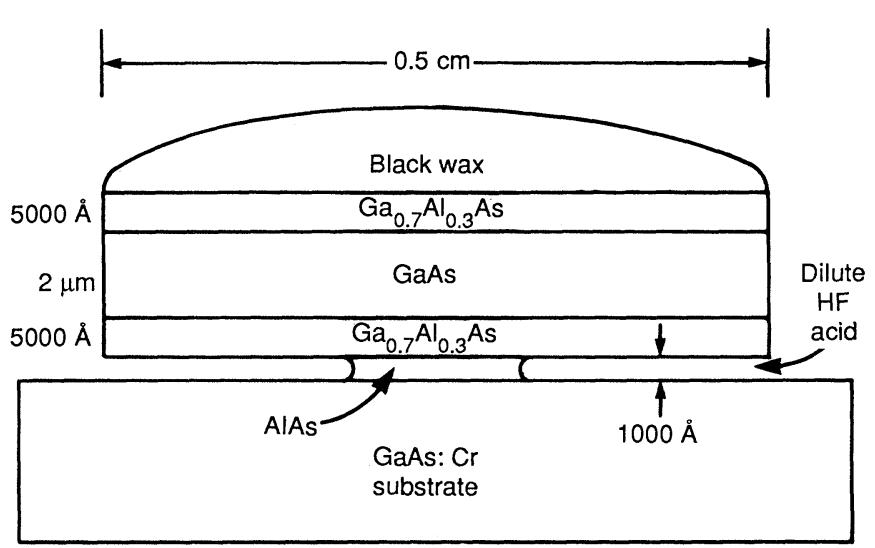

Fig. 4. A macroscopic multilayer epitaxial film is undercut by dilute HF. The AlAs release layer is shown as $1000 \AA$ thick but has been as thin as $20 \AA$ Epitaxial films as large as $2 \mathrm{~cm}$ by $4 \mathrm{~cm}$, and as thin as $200 \AA$, have been lifted off. 
Fig. 5. The epitaxial liftoff films can be bonded directly to glass by natural intermolecular forces. A $1500 \AA$ thick $\quad \mathrm{Al}_{0.3} \mathrm{Ga}_{0.7} \mathrm{As} / \mathrm{GaAs} /$ $\mathrm{Al}_{0.3} \mathrm{Ga}_{0.7} \mathrm{As}$ double heterostructure on a glass slide appears transparent with a brownish tint. The length marker is $1 \mathrm{~cm}$.

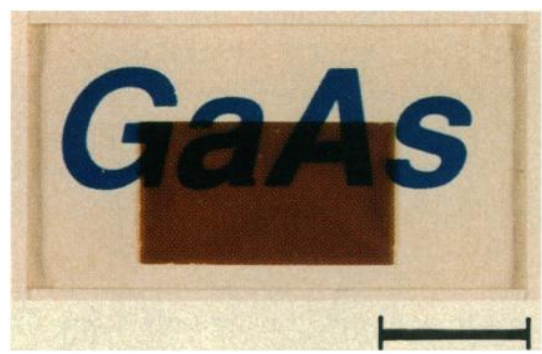

Thin-film epitaxy by OMCVD is more flexible, faster, lower in cost, and more suited for industrial production than MBE.

The development of double-heterostructure III-V semiconductor lasers (13) together with the demonstration of low-loss $\mathrm{SiO}_{2}$ glass fibers (14), which coincidentally occurred in the same year (1969), gave birth to optical communications. Optical fibers are already the technology of choice in new trunk and long-distance communications installations. It is predicted that optical fibers will eventually connect to individual telephone subscribers, making unprecedented bandwidth available for revolutionary new services.

The tremendous advance of solid-state electronics in the past $\mathbf{4 0}$ years was not inevitable, but depended on a combination of fortunate discoveries and on our ability to control chemical bonding structures, particularly at surfaces and interfaces. Progress was punctuated by three major accidents of inorganic surface (or interfacial) chemistry. (i) It all began fortuitously with the natural air-grown oxide of $\mathrm{Ge}$, which forms an interface with $99.99 \%$ saturated bonds. (ii) Almost 20 years later, the thermal oxidation recipe for $\mathrm{Si}$ was finally perfected. The $\mathrm{Si}-\mathrm{SiO}_{2}$ interface can have 99.9999\% saturated bonds. (iii) The accidental lattice match between GaAs and AlAs ensures an interface with $99.999 \%$ saturated bonds. The percentage of saturated bonds is the chemical figure-ofmerit for semiconductor interfaces.

\section{Current Applications of Semiconductor Surface Chemistry}

From these examples, and in hindsight, it is clear that our present level of electronic sophistication is the result of luck, trial-and-error, and the beneficence of nature, aided in small part by a few critical theoretical insights. It is much more difficult to predict what current work will be important in the future. I mention a few ongoing research topics, with disproportionate emphasis on my own research interests.

The search continues for semiconductor interfaces with ever better figures-of-merit. The best semiconductor interface now vn was identified as such only 3 or 4 years ago, though the zular surface has been well known since the dawn of the Si age. .. . .icrochip manufacturing plants all over the world, $\mathrm{Si}$ is oxidized thermally, and openings are etched in the oxide by hydrofluoric (HF) acid to expose the bare hydrophobic surface again. It is now apparent that this robust hydrophobic surface has a monolayer covering of predominantly $\mathrm{Si}-\mathrm{H}$ covalent bonds (15). In the most favorable of cases, this leaves less than one electrically active defect among $10^{7}$ surface atoms for a $99.99999 \%$ saturation of surface bonds, a record for any semiconductor interface (16). At present this is merely of scientific interest, since the $\mathrm{Si}_{-} \mathrm{SiO}_{2}$ interface is almost as good, and, being a buried interface, it is much more durable.

The vacuum-semiconductor interface has been the subject of a tremendous concentration of scientific effort in the past 15 years. Such surfaces are relatively easy to prepare, and a wide range of spectroscopic techniques are available to study them. Each different crystallographic orientation of a given crystal has its own plethora of surface reconstructions (local geometrical changes associated with surface-energy minimization). For example, the $(7 \times 7)$ reconstruction of the $\mathrm{Si}\langle 111\rangle$ surface has been studied in detail (17). Generally, vacuum-semiconductor interfaces do not fare well in terms of the chemical bond defect density figure-of-merit. A vacuum simply does not provide strong covalent bonding partners. The real importance of vacuum-semiconductor interface studies will probably lie in helping to understand and control III-V epitaxial growth processes (18). Ultimately, in order to fully exploit band-structure engineering and designer wave functions, an inorganic synthesis technology must be perfected such that the exact positions of the column III and column V elements in the final structure can be precisely controlled. In vapor phase growth, the surface reconstructions represent the chemical intermediate state, before the permanent tetrahedral structure is locked into place.

Direct growth of III-V epitaxial films on foreign substrates has also attracted considerable attention. Since the film and substrate material are not lattice matched, semiconductor quality is compromised. A new technology called epitaxial liftoff is expected to overcome these problems and find many uses in thin film science. Among alloys of the III-V semiconductors, only the Al-rich alloys are attacked by $\mathrm{HF}$ acid. The etch rate selectivity of AlAs versus GaAs in dilute HF acid is at least $100,000,000$ to 1 . If a thin AlAs layer is the first layer to be grown in a multilayer epitaxial sequence (Fig. 4), then a large-area film can be undercut from its growth substrate by taking advantage of this remarkable selectivity. Crackfree epitaxial films as large as $2 \mathrm{~cm}$ by $4 \mathrm{~cm}$ and as thin as $200 \AA$ have been lifted off in this way. This type of liftoff had been attempted in the past $(19,20)$ but has become practical only recently $(21)$. The AlAs release layer shown in Fig. 4 is $1000 \AA$ thick, but $20 \AA$ thick (seven monolayers) AlAs release layers work equally well. Importantly, the original growth substrate is reusable.

The epitaxial liftoff film, supported by wax, bonds naturally by van der Waals intermolecular forces to any smooth substrate. The bond is permanent, and the wax can be rinsed away in trichloroethylene. The transparent appearance of a $1500 \AA$ thick $\mathrm{Al}_{0.3} \mathrm{Ga}_{0.7} \mathrm{As} /$ $\mathrm{GaAs} / \mathrm{Al}_{0.3} \mathrm{Ga}_{0.7}$ As double heterostructure bonded to glass by van der Waals forces is shown in Fig. 5. To date a number of devices have been demonstrated: double-heterostructure GaAs/AlGaAs thin-film diode lasers on glass (22), GaAs metal-semiconductor field effect transistors (MESFETs) on glass and $\mathrm{Si}(23)$, strained singlequantum well InGaAs/GaAs high electron mobility transistors (HEMTs) (24), and GaAs optical receivers integrated onto $\mathrm{LiNbO}_{3}$ waveguides. We have extended this technology to InP-based semiconductors (quarternaries) through the use of ultrathin $(20 \AA)$ pseudomorphic strained AlAs release layers.

The ability to separate the epitaxial growth substrate from the active thin film permits a considerable increase in the sophistication of materials engineering. In particular, the properties of the supporting substrate can be optimized separately from those of the epitaxially grown film. There are many attributes in which III-V growth substrates have inadequate physical properties, among them being: radiation hardness for military electronics; mechanical strength; mechanical flexibility; thermal conductivity for high-power applications; light weight for space applications; substrate transparency for photomultiplier photocathode applications; low substrate dielectric constant for increased speed in supercomputers; low cost for solar cell applications; opto-electronic integration, such as III-V films on silicon; electro-optic integration, that is, III-V films on $\mathrm{LiNbO}_{3}$ and glass optical waveguides. Materials engineers can now combine the III-V devices to substrates that have the required physical properties.

Of these applications, the most interesting may be solar cells. It has been believed that there was an inevitable compromise between 
solar cell efficiency and cost. GaAs double heterostructures can be used to fabricate $30 \%$ efficient solar cells, and the epitaxial liftoff process allows them to be grown by OMCVD at low cost on reusable substrates. The economic barriers against large scale photovoltaic energy are probably severe enough that both high efficiency and low cost are necessary.

\section{Conclusions}

Most of the important surface chemistry on semiconductors is poorly understood. Particularly striking is the contrast between our detailed knowledge of semiconductor interfaces synthesized in vacuum and our poor understanding of those synthesized under ambient conditions. For example, the vacuum-prepared $\operatorname{Si}\langle 111\rangle(7 \times 7)$ is known in detail, but it has only recently become obvious that hydrophobic Si prepared by HF is covered with $\mathrm{Si}-\mathrm{H}$. Why is the silicon covered with $\mathrm{Si}-\mathrm{H}$ rather than the more thermodynamically stable $\mathrm{Si}-\mathrm{F}$ ?

The unexpected aspect of epitaxial liftoff is the immense etching selectivity between AlAs and GaAs of greater than $10^{8}$ to 1 . It comes as no surprise that AlAs etches in acids. The real mystery is the resistance of GaAs surfaces to HF. As an upper limit, GaAs appears to etch no faster than $1 \AA$ per hour in HF. No doubt, a particularly stable surface chemical structure develops on the GaAs, but we do not know its composition.

The explanation for the near total saturation of chemical bonds at the thermally grown $\mathrm{Si}_{-} \mathrm{SiO}_{2}$ interface is also still controversial. Part of the reason for our ignorance is that the defective chemical bonds are few and far between and therefore very difficult to study. But there are some other surfaces, particularly among the III-V semiconductors, for which even the major surface chemical constituent is unclear. There would appear to be an opportunity for multiple surface reflection infrared spectroscopy to help answer some of these basic questions.

The inorganic chemistry of semiconductors has been emphasized in this article. Organic chemistry has other important roles, but not as the active electronic material. Photoresists, the photographic materials that allow the patterning of microcircuits, were not discussed. Nor were the organic insulators, such as the polyimides, which are helpful in microcircuit packaging.
Control over the inorganic chemistry of semiconductor surfaces and interfaces has determined our rate of progress in solid-state electronics. A critical figure-of-merit is the concentration of defective chemical bonds at an interface, a defective chemical bond being defined as one that leaves an energy level in the forbidden gap. Our present level of technological advancement is the result of luck, trialand-error, and the beneficence of nature. Theoretical understanding has helped identify favorable chemical bonding structures when they have occurred and has shown us how to take advantage of them to create the revolutions in computers and communications that have defined the technology of our age.

\section{REFERENCES AND NOTES}

1. W. Shockley, Electrons and Holes in Semiconductors (Van Nostrand Reinhold, New York, 1950).

2. In present-day semiconductor lasers, $E_{\mathrm{Fv}}$ does not extend all the way down into the valence band as shown in Fig. 1, penalizing efficiency; see E. Yablonovitch and E. O. Kane, IEEE J. Lightwave Technol. LT-4, 504 (1986).

3. R. N. Hall, Phys. Rev. 87, 387 (1952).

4. W. Shockley and W. T. Read, ibid., p. 835.

5. M. M. Atalla, E. Tannenbaum, E. J. Scheibner, Bell Syst. Tech. J. 38, 749 (1959).

6. B. E. Deal, E. H. Snow, A. S. Grove, Semicond. Prod. Solid State Technol. 9, 25 (1966).

7. A. S. Grove, Physics and Technology of Semiconductor Devices (Wiley, New York, 1967).

8. Z. I. Alferov and R. F. Kazarinov, U.S.S.R. Inventor's Certificate no. 181737 (1963); H. Kroemer, Proc, IEEE 51, 1782 (1963)

9. M. A. Tischler, N. G. Anderson, S. M. Bedair, Appl. Phys. Lett. 49, 1199 (1986).

10. G. C. Osbourn, R. M. Biefeld, P. L. Gourley, ibid. 41, 172 (1982).

11. M. Tsuchiya et al., Phys. Rev. Lett. 62, 466 (1989).

12. F. L. Carter, Ed., Molecular Electronic Devices (Dekker, New York, 1982).

13. M. B. Panish and I. Hayashi, Appl. Phys. Lett. 16, 326 (1970).

14. R. D. Mauer, Proc. IEEE 61, 452 (1973).

15. V. A. Burrows, Y. J. Chabal, G. S. Higashi, K. Raghavachari, S. B. Christman, Appl. Phys. Lett. 53, 998 (1989).

16. E. Yablonovitch, D. L. Allara, C. C. Chang, T. Gmitter, T. B. Bright, Phys. Rev. Lett. 57, 249 (1986)

17. R. J. Hamers, R. M. Tromp, J. E. Demuth, ibid. 56, 1972 (1986).

18. D. E. Aspnes et al., ibid. 61, 2782 (1988).

19. F. Stern and J. M. Woodall, J. Appl. Phys. 45, 3904 (1974).

20. M. Konagai, M. Sugimoto, K. Takahashi, J. Cryst. Growth 45, 277 (1978).

21. E. Yablonovitch, T. Gmitter, J. P. Harbison, R. Bhat, Appl. Phys. Lett. 51, 2222 (1987).

22. E. Yablonovitch, E. Kapon, T. J. Gmitter, C. P. Yun, R. Bhat, IEEE Phot. Technol. Lett. 1, 41 (1989).

23. C. Van Hoof, W. D. Raedt, M. Van Rossum, G. Borghs, Electron. Lett. 25, 136 (1989).

24. D. R. Myers, J. F. Klem, J. A. Lott, Tech. Dig. Int. Electron Devices Meet. (1988).

25. I thank D. E. Aspnes, M. Bowden, and R. Frantz for carefully editing the manuscript. 